\title{
Teleconsultation for Pediatric Patients With Type 1 Diabetes Mellitus During the COVID-19 Pandemic: Experience of a University Hospital in Brazil.
}

\section{YASMIN EUGENIA SANTANA ( $\square$ yasmineugenia17@hotmail.com )}

Universidade de São Paulo Hospital das Clínicas da Faculdade de Medicina de Ribeirão Preto: Universidade de Sao Paulo Hospital das Clinicas da Faculdade de Medicina de Ribeirao Preto https://orcid.org/0000-0002-5094-497X

\section{Thais Milioni Luciano}

Universidade de São Paulo Hospital das Clínicas da Faculdade de Medicina de Ribeirão Preto: Universidade de Sao Paulo Hospital das Clinicas da Faculdade de Medicina de Ribeirao Preto

\section{Ana Carolina Maia Teodózio}

Universidade de São Paulo Hospital das Clínicas da Faculdade de Medicina de Ribeirão Preto: Universidade de Sao Paulo Hospital das Clinicas da Faculdade de Medicina de Ribeirao Preto

\section{Amanda Maria Barradas Monteiro de Santana}

Universidade de Sao Paulo Hospital das Clinicas da Faculdade de Medicina de Ribeirao Preto

\section{Letícia Rodrigues Ramos}

Universidade de São Paulo Hospital das Clínicas da Faculdade de Medicina de Ribeirão Preto: Universidade de Sao Paulo Hospital das Clinicas da Faculdade de Medicina de Ribeirao Preto

\section{Luiza Siqueira Rabelo Bastos}

Universidade de São Paulo Hospital das Clínicas da Faculdade de Medicina de Ribeirão Preto: Universidade de Sao Paulo Hospital das Clinicas da Faculdade de Medicina de Ribeirao Preto

\section{Brena de Sá Cardoso}

Universidade de São Paulo Hospital das Clínicas da Faculdade de Medicina de Ribeirão Preto: Universidade de Sao Paulo Hospital das Clinicas da Faculdade de Medicina de Ribeirao Preto

\section{Marília Oliveira Barbosa Alves}

Universidade de São Paulo Hospital das Clínicas da Faculdade de Medicina de Ribeirão Preto: Universidade de Sao Paulo Hospital das Clinicas da Faculdade de Medicina de Ribeirao Preto

\section{Patricia Volpon Santos Atique}

Universidade de São Paulo Hospital das Clínicas da Faculdade de Medicina de Ribeirão Preto: Universidade de Sao Paulo Hospital das Clinicas da Faculdade de Medicina de Ribeirao Preto

\section{Mariana Teresa Alves Sarti}

Universidade de São Paulo Hospital das Clínicas da Faculdade de Medicina de Ribeirão Preto: Universidade de Sao Paulo Hospital das Clinicas da Faculdade de Medicina de Ribeirao Preto

\section{Soraya Lopes Sader}


Universidade de São Paulo Hospital das Clínicas da Faculdade de Medicina de Ribeirão Preto: Universidade de Sao Paulo Hospital das Clinicas da Faculdade de Medicina de Ribeirao Preto

\section{Sonir Roberto Rauber Antonini}

Universidade de São Paulo Hospital das Clínicas da Faculdade de Medicina de Ribeirão Preto: Universidade de Sao Paulo Hospital das Clinicas da Faculdade de Medicina de Ribeirao Preto

\section{Mônica Freire Stecchini}

Universidade de São Paulo Hospital das Clínicas da Faculdade de Medicina de Ribeirão Preto: Universidade de Sao Paulo Hospital das Clinicas da Faculdade de Medicina de Ribeirao Preto

\section{Rodrigo José Custódio}

Universidade de São Paulo Hospital das Clínicas da Faculdade de Medicina de Ribeirão Preto: Universidade de Sao Paulo Hospital das Clinicas da Faculdade de Medicina de Ribeirao Preto

\section{Raphael Del Roio Liberatore Junior}

Universidade de São Paulo Hospital das Clínicas da Faculdade de Medicina de Ribeirão Preto: Universidade de Sao Paulo Hospital das Clinicas da Faculdade de Medicina de Ribeirao Preto

\section{Research}

Keywords: diabetes, teleconsultantion, COVID-19

Posted Date: April 13th, 2021

DOI: https://doi.org/10.21203/rs.3.rs-404736/v1

License: (1) This work is licensed under a Creative Commons Attribution 4.0 International License. Read Full License

Version of Record: A version of this preprint was published at Jornal de Pediatria on March 1st, 2022. See the published version at https://doi.org/10.1016/j.jped.2022.02.001. 


\section{Abstract}

Background: The SARS-CoV-2 pandemic expanded rapidly around the world in 2020 and health services needed to be reconfigured to meet the new demand and ensure the care of patients with chronic diseases, whether or not aggravated by this COVID-19 (disease caused by SARS-Cov-2). For patients with chronic diseases - such as diabetes mellitus - the teleconsultation stood out as a tool for clinical management in this period. This study aims to evaluate the impact of teleconsultation on glycemic control and prevention of acute complications related to diabetes mellitus in children and adolescents treated by the pediatric endocrinology team at Hospital das Clínicas, Ribeirão Preto Medical School, University of São Paulo (HC-FMRP -USP) during the COVID-19 pandemic in 2020.

Method: Descriptive study of data from pediatric diabetic patients who received teleconsultation via messages in the months of April to September 2020.

Results: Regarding the patients evaluated $(\mathrm{N}=143)$, there were 3.4 teleconsultations per patient in the studied period; requiring adjustment of insulin doses in $84.6 \%$ of cases. The hospital admission rate was $17.5 \%$ due to diabetic decompensation and / or the opening of diabetes mellitus. The dosage of glycosylated hemoglobin showed a worsening in $46 \%$ of the sample and an improvement in $37 \%$.

Conclusion: The teleconsultation was effective in ensuring health care for patients with DM1 during the COVID-19 pandemic.

\section{Background}

The outbreak of severe acute respiratory syndrome resulting from infection with the new coronavirus (SARS-CoV-2) started in China in late 2019 spread rapidly across the world and was officially recognized as a pandemic by the World Health Organization in March $2020^{1}$. Until at the time of writing this manuscript (March / 2021), there are 117,764,619 infected with the coronavirus in the world and more than 2 million deaths ${ }^{2}$. The United States leads in number of infected and dead, with Brazil in second place in number of dead and in third place in number of infected in the world ${ }^{2}$. The first confirmed case of the new coronavirus infection in Brazil occurred on February 26, 2020, in the city of São Paulo ${ }^{3}$. Ribeirão Preto had its first confirmed case on March 21, 2020 and, until March 9, 2021, it has 55,857 confirmed cases, according to an epidemiological bulletin released on March $10,2021^{5}$.

According to the Brazilian Diabetes Society, it is estimated that in Brazil 7.6 per 100 thousand inhabitants under the age of 15 are carriers of type 1 diabetes mellitus (T1DM), with an increase in this incidence being observed in the last decades, particularly in children under 5 years ${ }^{9}$. T1DM is a chronic disease, requiring multidisciplinary follow-up and regular medical visits for education, treatment adjustment and screening for associated comorbidities. However, due to the current context of the pandemic, the pediatric endocrinology scientific committee of the Brazilian Society of Pediatrics published a warning note on April 7, 2020 on DM1 and COVID-19 (disease caused by the new coronavirus) in pediatrics. It did not 
recommend elective consultations to avoid the risk of contamination and encouraged contact with the medical team by phone, text messages and e-mails ${ }^{10}$.

The WHO defines telemedicine as "the provision of health services, where distance is a critical factor, by all health professionals, using information and communication technologies to exchange valid information for the diagnosis, treatment and prevention of diseases and injuries, for research and evaluation, and for the continuing education of health care providers, all in the interest of promoting the health of individuals and their communities " 8 .

National and local determinations authorized the use of telemedicine, allowed the HC-FRMP-USP child endocrinology team to reduce ambulatory activities to what is strictly necessary, transferring the majority of consultations to telemedicine and maintaining hospitalizations only on an urgent basis.

This type of assistance had never been carried out on a large scale. With the need for new forms of medical care, associated with the large-scale dissemination of a new virus, it is not known what was the impact of the use of new modalities of medical care in the clinical control of patients.

\section{Methods}

This study aimed to assess the impact of call center on clinical follow-up and metabolic control in pediatric diabetic patients during the COVID-19 pandemic.

A descriptive study on teleconsultations of diabetic patients who are followed up at the HC-FMRP-USP was carried out from April to September 2020, during the COVID-19 pandemic.

Data were collected from the review of text messages made via the application program Whats App ${ }^{\circledR}$ through the exchange of messages between the medical team and those carers of patients. The data were organized in an Excel ${ }^{\circledR}$ spreadsheet. The teleconsultation consisted of those that included: sending glycemic controls to the conference by the assistant team; checking the insulin doses in use; verification of the presence of symptoms of diabetes decompensation; adequacy of insulin doses. The number of telephone calls made in the period; the need to adjust insulin doses; the need for hospitalization due to diabetes decompensation or for opening the picture and the comparison of glycosylated hemoglobin values before and during the COVID-19 pandemic were evaluated in this study.

This study was submitted to the Research Ethics Committee of HC-FRMP-USP and approved via Plataforma Brasilß (No. 4.220.266).

\section{Results}

This study included 170 pediatric patients with T1DM, followed at HC-FMRP-USP, who made telephone contact with the pediatric endocrinology team during the period from April to September 2020. Patients $(n=27)$ who came in telephone contact were excluded just to clear up doubts, request exams and / or 
renew prescriptions and schedule an outpatient return. The epidemiological characteristics of the 143 study participants are summarized in Table 1 . There was a predominance of females (57\%) over males. There was also a predominance of the adolescent age group (59\%) in the patients evaluated. Of the patients evaluated, 13 were new cases and $30 \%$ had less than 2 years of diagnosis of T1DM.

Table 1

Description of diabetic patients followed by teleconsultation at HCFMRP-USP.

\begin{tabular}{|ll|}
\hline Characteristics & $\begin{array}{l}\text { Number of patients } \\
\text { (\% in relation to total }-\mathbf{n}=143)\end{array}$ \\
\hline Sex & $82(57)$ \\
\hline - Female & $61(43)$ \\
\hline - Male & \\
\hline Age & $4(3)$ \\
\hline - Infant (0-2 years) & $17(12)$ \\
\hline - Early Childhood (2-7 years) & $37(26)$ \\
\hline - Middle Childhood (7-11 years) & $85(59)$ \\
\hline - Adolescence (11-20 years) & \\
\hline Diagnosis time of T1DM & $13(9)$ \\
\hline - New cases & $44(30)$ \\
\hline - 0-2 years of diagnosis & $34(24)$ \\
\hline - 2-5 years of diagnosis & $32(22)$ \\
\hline$-5-10$ years of diagnosis & $11(15)$ \\
\hline - More than 10 years of diagnosis & \\
\hline
\end{tabular}

According to the glycemic control data sent, adjustments were made to the doses of medications in use and the prescriptions (digitalized or printed) were updated. 493 teleconsultations were carried out for the 143 pediatric diabetic patients included in the study, with an average of 3.4 teleconsultations per patient in the evaluated period (5 months).

The period of use of the application for teleconsultations was, on average, 40 hours per week, totalizing a period of approximately 1040 hours.

Due to the glycemic controls evaluated in the teleconsultations, it was necessary to adjust the insulin doses in $84.6 \%$ of the attendance. Patients who required high doses of insulin in adjustments, even after 
reinforcement of the appropriate technique of application and correct handling of insulin, as well as those whose glycemic controls remained altered despite the adjustments of insulin therapy, were summoned to personal assistance and reassessment of the case.

Of the 143 patients evaluated, $17.5 \%$ needed hospitalization during the study period; sometimes due to the onset of diabetes mellitus (13 cases), sometimes due to the decompensation of the disease (12 cases). There was no need for more than one hospital stay in the evaluated period.

Regarding metabolic control, the glycosylated hemoglobin values of pediatric diabetic patients were compared before and during the COVID-19 pandemic in 2020. This test represents the global weighted average of the mean daily blood glucose levels during the last two to three months ${ }^{11}$. The glycosylated hemoglobin values of pediatric diabetic patients were compared before and during the COVID-19 pandemic in the year 2020. In order to compare the values before and during the pandemic, those who presented a drop in glycosylated hemoglobin value greater than $0.5 \%$ were considered as improvement; those who presented an increase in glycosylated hemoglobin value greater than $0.5 \%$ were considered as worsening; values that showed a percentage difference of less than $0.5 \%$ of glycosylated hemoglobin were considered as maintained. Among the 67 patients who underwent the examination at both moments of evaluation, $46 \%$ showed worsening, $37 \%$ showed improvement and $17 \%$ showed maintained value of glycosylated hemoglobin during the COVID-19 pandemic. Of these patients, $30 \%$ had glycated hemoglobin within the therapeutic target $(<7.5 \%)^{11}$.

The evolution of these glycosylated hemoglobin values was also compared to the previous evolution of this test in the same patients $(n=67)$ before the pandemic during 2019, as described in Table 2 .

Table 2

Comparison of glycosylated hemoglobin values before and during the COVID-19 pandemic.

\begin{tabular}{|ll|}
\hline \multicolumn{2}{|l|}{ Comparison of glycosylated hemoglobin values $(\mathbf{n}=\mathbf{6 7})$} \\
\hline Before the COVID-19 pandemic (2019) & During the COVID-19 pandemic (2020) \\
\hline Improvement 36\% & Improvement $37 \%$ \\
\hline Worsening 34\% & Worsening $46 \%$ \\
\hline Maintained 30\% & Maintained $19 \%$ \\
\hline
\end{tabular}

\section{Discussion}

The teleconsultation in the diabetic population was already an important strategy for monitoring, even before the current pandemic, evidencing adequate glycemic control of patients undergoing this form of clinical care ${ }^{13}$. Considering the need for adequate follow-up of these patients in the current pandemic context, in which social isolation is mandatory for the prevention of COVID-19, this form of care must be considered and evaluated in order to guarantee the best possible health care for patients. 
In comparison to the attendance that was previously carried out, the number of teleconsultations exceeds what was commonly performed. During the follow-up of pediatric diabetic patients at HC-FMRP-USP, returns were scheduled every 2-3 months for clinical reevaluation and periodic examinations (on average, 2 visits in a period of 5 months). The time taken by the teleconsultations exceeds 5 times the time usually devoted to personal assistance for pediatric diabetic patients at HC-FMRP-USP (8 hours per week; 208 total hours in 5 months). It was then possible to identify that the time spent on care for patients and their carers was higher, which allowed adjustments to insulin doses and reinforced the T1DM care guidelines more frequently.

A recent publication by the Barbara Davis Center reports success in glycemic control of new cases of $\mathrm{T}_{1} \mathrm{DM}^{15}$. Patients diagnosed during the pandemic had their education in diabetes and management of insulin doses made exclusively by telemedicine after hospital discharge. A combination of e-mail, video calls and telephone calls was used to provide guidance, check blood glucose levels and adjust the medication of these patients, achieving good glycemic control at the end of the reported period, without the need for personal assistance by health services.

The rate of hospitalization found occurred in a similar way, both due to the opening of T1DM cases and to the decompensation of this disease. There was an adjustment of insulin doses, review of the application technique, as well as correction of any errors regarding the care of the disease during hospitalization of these patients. In addition to the health care already provided to hospitalized patients before the COVID-19 pandemic, teleconsultations were also carried out after hospital discharge, which contributed to the avoidance of rehospitalization in the period from April to September 2020 for patients with DM1.

Despite the predominance of worsening glycosylated hemoglobin values during the COVID-19 pandemic, it was also possible to identify an improvement in values. The improvement in glycemic control by telemedicine and the use of a messaging application has also occurred in other studies. A systematic review found that diabetic patients monitored by telemedicine showed a small, but statistically significant, reduction in glycosylated hemoglobin compared to patients who were exclusively monitored by conventional medical care $(-0.31 \%, p<0.001)^{13}$. A study conducted by a group in Turkey sought to assess the impact of the teleconsultations carried out by a multidisciplinary team (doctor, nurse, nutritionist and psychologist) and it was observed that those patients who used the service more frequently (daily every fortnight) showed an improvement mean glycated hemoglobin $(-0.85 \%, p<0.01)$ compared to those who used the service less regularly ${ }^{13}$.

The rate of improvement of glycated hemoglobin and even those who showed values within the therapeutic target in this study demonstrate that this new form of patient care may be promising in the future of medicine.

\section{Conclusion}


Due to the need for a significant reduction in the number of face-to-face outpatient consultations, in order to guarantee an effective social distance, necessary due to the pandemic by COVID-19, telemedicine provided guidance and assistance to patients. This new form of medical care provided adequate attention to the health of pediatric diabetic patients followed by the Pediatric Endocrinology team at HCFRMP-USP. Our experience with the teleconsultations was positive and proved to be promising for the clinical follow-up of cases of patients with chronic diseases, such as those with T1DM in the pediatric age group.

\section{Abbreviations}

COVID-19: disease caused by the new coronavirus SARS-Cov-2.

HC-FMRP-USP: Hospital das Clínicas, Ribeirão Preto Medical School, University of São Paulo.

T1DM: type 1 (one) of diabetes mellitus.

\section{Declarations}

\section{Ethics approval and consent to participate:}

This study was approved by Research Ethics Committee of HC-FRMP-USP via Plataforma Brasil $\AA^{\text {(No. }}$ 4.220.266).

\section{Consent for publication:}

Not applicable.

\section{Availability of data and materials:}

All data generated or analysed during this study are included in this published article.

\section{Competing interests:}

The authors declare that they have no competing interests.

\section{Funding:}

Own funding.

\section{Authors' contributions:}

Not applicable.

\section{Acknowledgements:}


Not applicable.

\section{References}

1. Mahase E. Covid-19: WHO declares pandemic because of alarming levels of spread, severity, and inaction. BMJ. 2020; doi: https://doi.org/10.1136/bmj.m1036.

2. Johns Hopkins University and medicine. Coronavirus Resource Center. http://coronavirus.jhu.edu/map.html. Accessed 10 March 2021.

3. Brazil. Ministry of Health. Coronavirus panel. https://covid.saude.gov.br. Accessed 10 March 2021.

4. São Paulo. Health Secretariat of São Paulo. Distribution of population, cases and deaths. http://seade.gov.br/coronavirus. Accessed 10 March 2021.

5. Ribeirão Preto City Hall. Health Secretariat - Epidemiological Surveillance Division. Coronavirus epidemiological bulletin (COVID19). https://www.ribeiraopreto.sp.gov.br/portal/saude/boletim-novocoronavirus-covid-19. Accessed 10 March 2021.

6. Federal Council of Medicine. CFM Official Letter No. 1756/2020 - COJUR. 2020. https://portal.cfm.org.br/images/PDF/2020_oficio_telemedicina.pdf. Acessed 10 March 2021.

7. Law n 13,989, of April 15, 2020. Diario Oficial da União. 2020.

https://www.in.gov.br/en/web/dou/-/lei-n-13.989-de-15-de-abril-de-2020-252726328. Acessed 10 March 2021.

8. WHO Global Observatory for eHealth. Telemedicine: opportunities and developments in Member States: report on the second global survey on eHealth. World Health Organization. . https://apps.who.int/iris/handle/10665/44497. Acessed 10 March 2021.

9. Brazilian Diabetes Society. Guidelines of the Brazilian Diabetes Society 2019-2020. https://www.diabetes.org.br/profissionais/images/DIRETRIZES-COMPLETA-2019-2020.pdf. Acessed 10 March 2021.

10. Alves, CAD, et al. Alert note: Diabetes mellitus and COVID-19 in Pediatrics. Brazilian Society of Pediatrics. 2020. https://www.sbp.com.br/fileadmin/user_upload/22440b-NA__Diabetes_Mellitus_e_COVID-19_em_Pediatria.pdf. Acessed 10 March 2021.

11. Brazilian Diabetes Society. Guidelines of the Brazilian Diabetes Society 2014-2015. Update on glycated hemoglobin: clinical manifestations. 2015.

https://www.diabetes.org.br/publico/images/2015/area-restrita/diretrizes-sbd-2015.pdf. Acessed 10 March 2021.

12. Flodgren $\mathrm{G}$, et al. Interactive telemedicine: effects on professional practice and health care outcomes. Cochrane Database of Systematic Reviews. 2015; doi: 10.1002 / 14651858.CD002098.

13. Timpel $P$, et al. Mapping the evidence on the effectiveness of telemedicine interventions in diabetes, dyslipidemia, and hypertension: an umbrella review of systematic reviews and meta-analyzes. J Med Internet Res. 2020; doi: 10.2196 / 16791. 
14. Doger $E$, et al. Effect of telehealth system on glycemic control in children and adolescents with type 1 diabetes. J Clin Res Pediatr Endocrinol. 2019; doi: 10.4274 / jcrpe.galenos.2018.2018.0017.

15. Garg SK, Rodbard D, Hirsch IB, Forlenza GP. Managing New-Onset Type 1 Diabetes During the COVID19 Pandemic: Challenges and Opportunities. Diabetes Technol Ther. 2020; doi:

10.1089/day.2020.0161. 